\title{
Implementation of SAAS Compiler in Intranet
}

\author{
V.Balu \\ Assistant Professor, \\ Department of CSE, SCSVMV University, \\ Enathur, Kanchipuram, Tamilnadu
}

\author{
M.Saraswathi \\ Assistant Professor, \\ Department of IT, SCSVMV University, Enathur, \\ Kanchipuram, Tamilnadu
}

\begin{abstract}
This paper information about the successful use of Webbased interface to $\mathrm{C}, \mathrm{C}++$ and Java compilers. There are several benefits that make networked software desirable. A web-based application can be used remotely access by the users throughout any network connection and making it platform independent by any operating system. There is no local installation and maintenance work necessary. This proposed system allows the users to use the software as a service(SAAS) feature to practice and do their works from wherever and they have access the network at whatever time. Virtualized software can be used over a network through browser to create a virtual laboratory which can be used by the clients at any place. An RSS (Rich Site Summary) ability to be added for allowing the users to know about the current events and also update everyone's location in real-time. A local mail system can be useful inside the network facilitating clients to clear queries with the staff or even other clients very easily. Real-time event collaboration can be done by adding a calendar which can be updated regularly to facilitate message broadcasting. This system achieves better utilization of the available resource. This proposed system allows users to use the virtualized software without installing in the client system.
\end{abstract}

Keywords:Compilers,Virtualized software,SAAS,Rich site memory.message broadcasting.

\section{INTRODUCTION}

Software as a Service (SaaS) is a software distribution model in which applications are hosted by a vendor or service provider and made available to customers over a network, typically the Internet[1]. SaaS is becoming an increasingly prevalent delivery model as underlying technologies that support web services and service-oriented architecture (SOA) mature and new developmental approaches like Ajax. Meanwhile, broadband service has become increasingly available to support user access from more areas around the world. The hosted application management (hosted AM) model is similar to Active server pages (ASP) technology, the Asp provider hosts software commercially for customers and delivers it over the Web. In the software on demand model, the provider gives to a single copy of an application created specifically for SaaS distribution on network-based access. SaaS model includes easier administration, automatic updates and patch management, compatibility, all users will have the same version of software, easier collaboration, global accessibility.But in the traditional model of software distribution, in which software is purchased for and installed on personal computers, is sometimes referred to as software as a product. Intranet is the generic term for a collection of private computer networks within an organization. An intranet uses network technologies as a tool to facilitate communication between people or work groups to improve the data sharing capability and overall knowledge base of an organization's employees.

\section{RELATED WORK}

Software as a Service is a software distribution model in which applications are hosted by a vendor or service provider and made available to customers over a network, typically the Internet. Now a days, SAAS is used in intranet using a cloud server with in a organization. In this proposed system software will be packed with a Document Viewer and a Compiler. From this we can get more benefit such as users need not install software such as a Document Viewer or a Language compiler in their system, more control in the intranet, security, timely backups, better utilization of Unused Server clock, collaboration of Events over a calendar, information about occurrences in an RSS Feed. The Objective of this system is to implement SaaS (without an active internet connection) in Intranet.

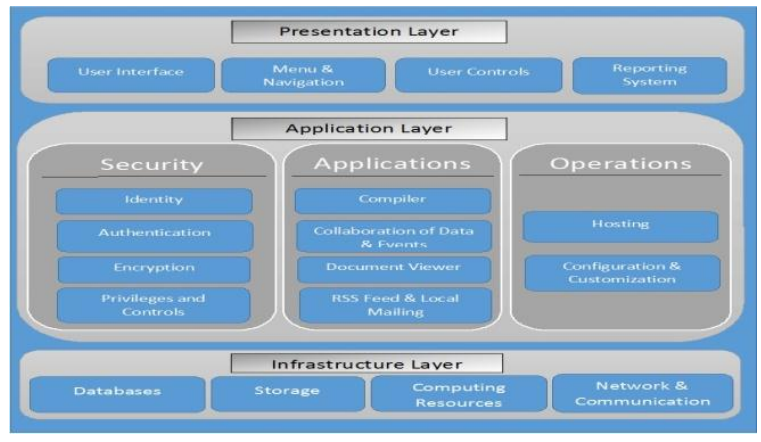

Fig 1. System Architecture

Architecture consists of three layers are infrastructure, Application and presentation layer. Infrastructure layers provide the technical capabilities required for storing data and moving it around the network. Application layer represent the typical business layer or middle tier of a SaaS application. Operations are the capabilities needed to efficiently keep the SaaS application running. Security is one of the most important categories of SaaS capabilities, given that Intel's data and user accounts are typically hosted by the SaaS provider. Presentation layer includes all capabilities exposed to the user, such as Menu and navigation. These provide access to the features and functionality within an application, organized in an intuitive way so that the user can select the desired function. Application-specific predefined or ad-hoc reports.

The proposed system gives easy way to compile, view file and convert to another format. It provides benefits such as no installation of software, no license cost, less memory 
utilization etc.The main reason for creating this system to provide a centralized compiling scheme. Also, it will act as a centralized repository for all the codes written. The other major advantage of this system is no need to maintain separate compilers at the client side.

\section{TECHNOLOGY USED}

In this system using IIS to Host the Document Viewer developed in ASP.Net. IIS(Internet information Service) is used in Microsoft servers to run many web services in Windows Environment, it was first released with Windows NT. IIS supports HTTP,HTTPS, FTP, SMTP etc.JDK is required to compile and run Java programs. JDK installed in the Ubuntu Machine, the Ubuntu machine will be the house of the Apache server. The Ubuntu machine will do the compilation of the codes which are typed in the browser using the JDK installed in it.The office suite is used to convert the document files to pdf inside the server, which in turn can be opened by the browser. The backend or the database is created using MySQL database. MySQL Database will work with the AMPPS Stack, the database comes with a Graphical Interface which will allow the administrator to take care of the database using Queries or using the User Interface.

Rich Site Summary(RSS) is originally RDF Site Summary, is a format for delivering regularly changing web content. Many news-related sites, weblogs and other online publishers syndicate their content as a RSS Feed to whoever wants it. An RSS document called "feed", "web feed", or "channel" includes full or summarized text, and metadata, like publishing date and author's name. Extension of filename is.rss, .xml[3].

\section{IMPLEMENTATION}

In this system developed several modules such as UI Design, User database, Login forms, Admin User database, Language Compilers, Document viewer, Intranet mailing.

\section{- UI Design}

The front end or the User Interface will comprise of the User login form, the admin login form, Admin profile, user profiles, Navigation through the web application. The compiler interface will also be a sub module in the front end or the User Interface. The whole user interface has been created using the Bootstrap framework. The interface is completely responsive and will take the correct structure of the page when the browser is resized.

\section{- User database}

User database stores the information about the users who have registered for the service. It also stores the information such as usernames and passwords of the active user. The passwords stored in the database are encrypted for the security and privacy of the users.

\section{- Login Forms}

Login forms are required for the users and the administrators to login to the application, the username and passwords are collected from the login form and checked with the database and authenticated. There are two types of accounts the normal user and the administrators with increased privileges.

\section{- Admin User Interface}

The admin interface will be different from the normal users' interface. The administrator accounts will have privileges to open and look into the database and also delete user accounts and information from the database.

\section{- Language Compilers}

The main aim of the system is to virtualized Language compilers, the most used languages in the University campus are $\mathrm{C}, \mathrm{C}++$ and Java. The compiler works on a Networked Environment, users having access to the intranet can use these compilers without installing any software on their client systems.

\section{- Document Viewer}

The Document Viewer allows users to read or view documents such as Word documents, Excel Spreadsheets, Rich Text Files and also other documents. It allows users to upload documents through the browser and view the documents. The application converts the uploaded documents to pdf and returns it to the browser, which can be read easily.

\section{- Intranet Mailing}

The intranet mailing feature allows users to send EMail using the Intranet to users who have registered for this application. The intranet mail has been set up using an Open Source system. It uses the Squirrel Mail system which is an open source project and is a very stable mailing function.

\section{EXPERIMENTAL RESULTS}

The compiler is centralized and allows the users to execute programs as per the requirements of the students or the programmers. When a user enters programs in the text field given the code is sent to the server and is executed inside the server's environment. The output of the code is posted in the output text field, errors if any are sent to the errors text field in the form of text from the server in which the execution of the code takes place. .The snapshots of Java Compiler and its output are displayed in figure 2 and 3.

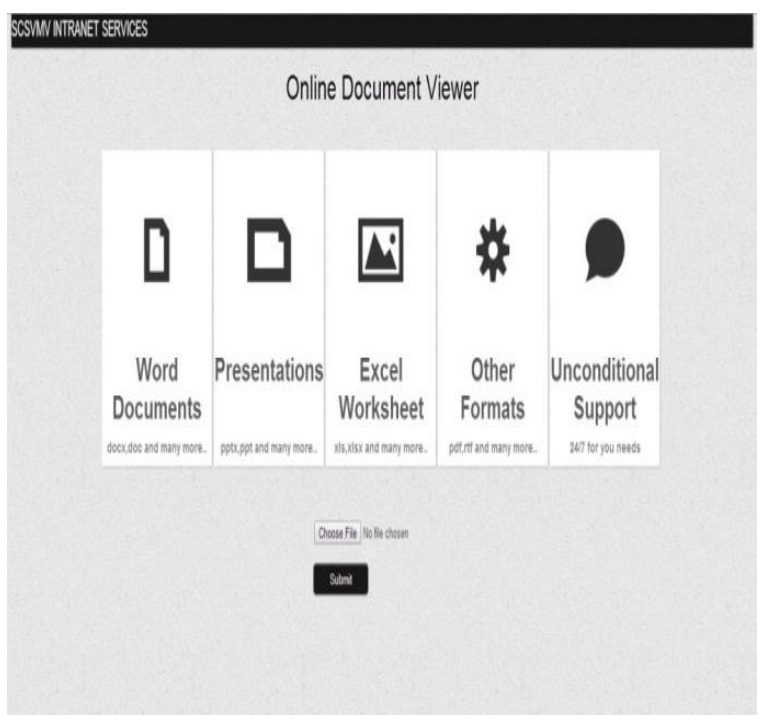

Fig 2. Java Compiler 


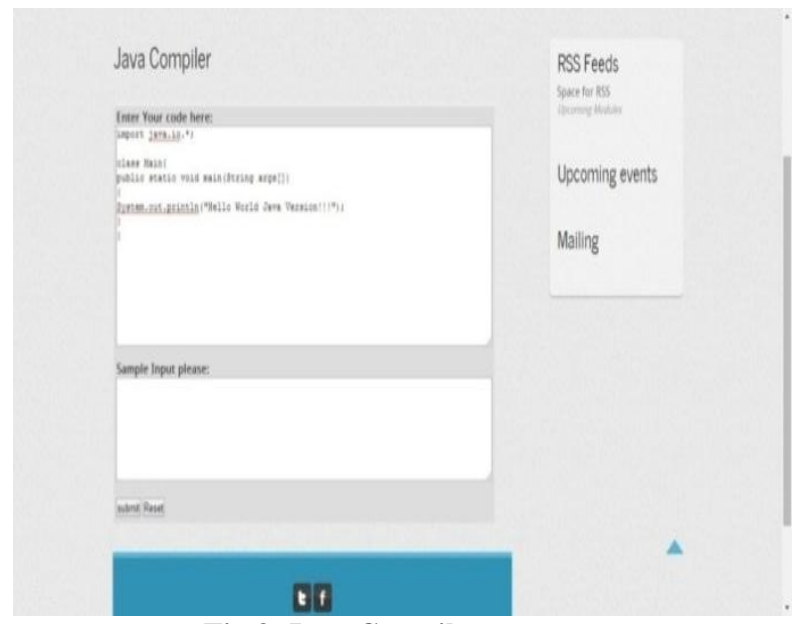

Fig 3. Java Compiler output

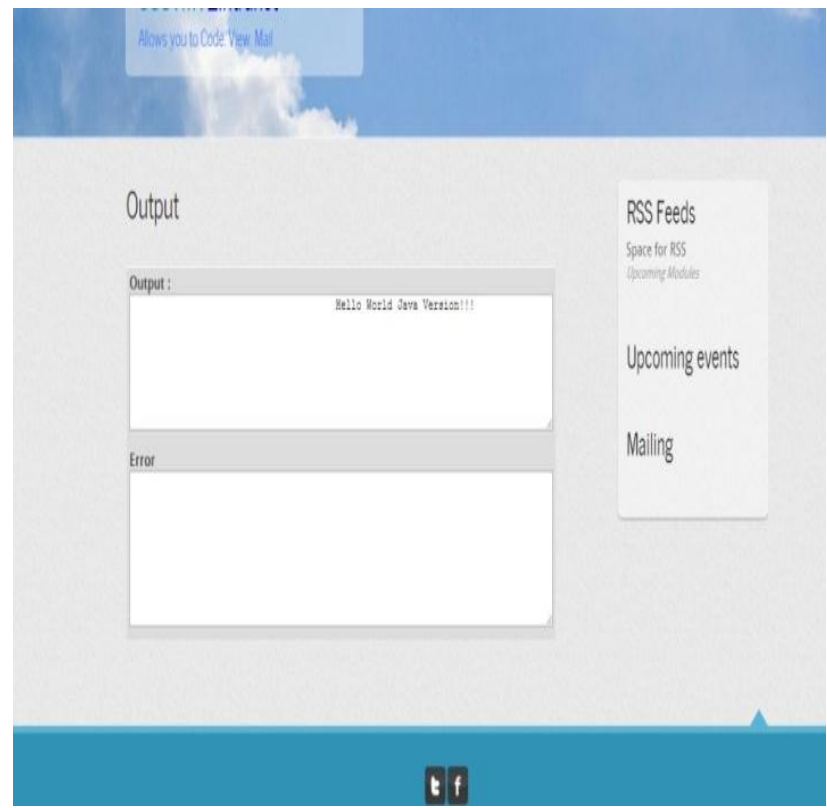

Fig 4. $\mathrm{C}, \mathrm{C}++$ Compiler

The snapshots of $\mathrm{C} \& \mathrm{C}++$ Compiler displayed in figure 4 When the user tries to use the Document Viewer they are prompted to a home page where they can upload the document file to the server. In the server the document file is converted to pdf format, which is supported by any browser and can allow the user to view the documents such as Spreadsheets, Word Documents, PowerPoint Presentations, and also Rich Text. The snapshots of Document Viewer and its executed output represented in figure 5 and 6.

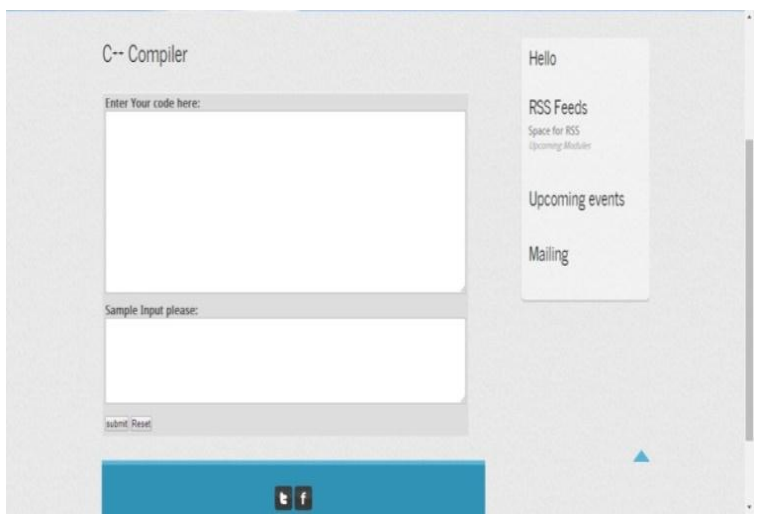

Fig 5.Document Viewer

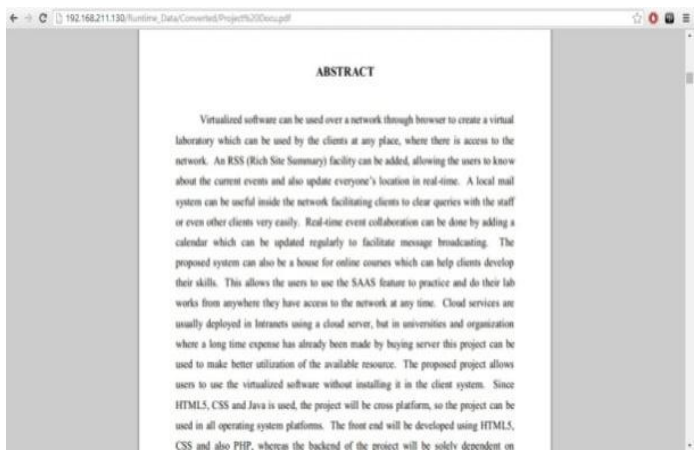

Fig 6.Document Viewer Output Screen

\section{CONCLUSION}

In this work, present a creating an online compiler system in the domain of Computer Networks is achieved. The compiler is centralized and allows the users to execute programs as per the requirements of the students or the programmers. The other major advantage of this system is no need to maintain separate compilers at the client side and converting document file into different types of formats.

\section{REFERENCES}

[1] http://searchcloudcomputing.techtarget.com/definition /Software-as-a-Service

[2] Aleksander Malinowski, Bogdan M. Wilamowski Bradley University, Peoria, IL / University of Wyoming, Laramie, WY,Web-based C++ Compiler, session 2532

[3] http://www.slideshare.net/renzilde/rss-28400890

[4] www.codeproject.com

[5] www.Stackoverflow

[6] Msdn.Microsoft.com 